\title{
Effects of production system and feeding regimen on carcass and meat quality traits of Naked Neck chicken
}

\author{
E. Bughio', J. Hussain ${ }^{1}$, A. Mahmud" \& A. Khalique ${ }^{2}$ \\ ${ }^{1}$ Department of Poultry Production, Faculty of Animal Production and Technology, University of Veterinary and Animal \\ Sciences, Lahore, 54000, Pakistan \\ ${ }^{2}$ Department of Animal Nutrition, Faculty of Animal Production and Technology, University of Veterinary and Animal \\ Sciences, Lahore, 54000, Pakistan
}

(Submitted 24 July 2020; Accepted 15 January 2021; Published 16 April 2021)

\begin{abstract}
To evaluate the effect of a production system and feeding regimen on meat quality attributes of Naked Neck chickens, a total of 150 cockerels at 18 weeks old $(1625 \pm 70 \mathrm{~g})$ were collected from 10 treatment groups with five replicates of three birds. The factorial arrangement of treatments consisted of two production systems (intensive and free-range) and five nutritional regimens, namely 100\% commercial feed; $75 \%$ commercial feed plus 25\% kitchen waste; $50 \%$ commercial feed plus 50\% kitchen waste; $25 \%$ commercial feed plus $75 \%$ kitchen waste; and 100\% kitchen waste. Carcass traits, meat quality, and meat organoleptic were found to differ significantly among production systems, feeding regimens, and their interaction. Higher liver weight was observed in birds reared under an intensive system. Higher gizzard weight was noted in birds fed with $100 \%$ kitchen waste, whereas lower gizzard weight was observed in birds fed the commercial diet. The meat from cockerels fed with $75 \%$ kitchen waste was most yellow, whereas the meat from the birds fed with $100 \%$ kitchen waste was least yellow. At two hours after slaughter, $\mathrm{pH}$ of the meat was highest in birds fed $50 \%$ kitchen waste and lowest in birds fed 100\% kitchen waste. The interaction of production system and feeding regimen was significant for overall acceptability score. In conclusion, Naked Neck chickens performed equally well under intensive and free-range systems, irrespective of the level of kitchen waste that they were fed.
\end{abstract}

Keywords: carcass, kitchen waste, nutritional regimes, sensory attributes

${ }^{\#}$ Corresponding author: atharmahmud@uvas.edu.pk

\section{Introduction}

Food security is a challenge globally because of the increasing human population and decreasing nutritional resources. Meat and eggs from chickens are considered the cheapest, most readily available source of quality protein for sustaining health and nutrition for this ever-increasing population (Ajayi, 2010; Sow \& Grongnet, 2010; Shahzad et al., 2011; Rahman et al., 2013; Mueller et al., 2018). In Pakistan, poultry production plays an essential role in bridging the divide between the demand for animal protein and its supply, and is a dynamic contributor to animal agriculture. Poultry meat accounted for 35 per cent (1657 thousand tons) of the meat produced in Pakistan in 2019 - 2020 (ESOP, 2020). The rural and commercial poultry sectors contributed about 124.72 and $1,531.60$ million metric tons to the total production of meat (ESOP, 2020).

Indigenous chickens that are well adapted to local environmental conditions with excellent resistance to endemic diseases play a significant role in food security and socio-cultural activities in the rural population (Yousif \& Eltayeb, 2011; Zhao et al., 2015; Padhi, 2016; Rajkumar et al., 2017). A wide variation of choice is observed, especially among urban populations, where some segments of society prefer meat from organic backyard-type chickens, whereas others prefer meat from commercial meat-type chicken genotypes, because less time is required for preparation and cooking. Such variation in preferences may serve as a threat to the local backyard-type chickens or as an opportunity for these chickens to be used more widely. 
The preference of consumers for meat products is affected strongly by sensory characteristics such as juiciness, flavour, and aroma (Chulayo et al., 2011; Jin et al., 2019). Carcass, physicochemical and sensory attributes of meat are affected significantly by diet in both commercial and indigenous chickens (Youssao et al., 2012). The nutritional profile of meat varies in different feeding and housing systems (Ying et al., 2011), live body and carcass weights, physicochemical traits, and sensory attributes (Wattanachant, 2008; Yousif \& Eltayeb, 2011; Olaniyi et al., 2012; Ojedapo, 2013; Ahmad et al., 2019). Recent findings supported freerange housing systems, which lead to more cooking and drip loss, which result in healthier food with less fat in comparison with meat from birds reared in the cage system (Xiang et al., 2018). Free-range rearing affects the birds' welfare, health, and production efficiency. Consumers prefer free-range products and are willing to pay a premium for them (Zhang et al., 2018). Products from free-range systems are generally healthier and have had higher welfare standards from a consumer standpoint than those from the conventional intensive systems (Rehman et al., 2017; Jin et al., 2019).

Backyard chickens are considered slow growing with relatively low carcass weight (Tougan et al., $2013 a, b)$. However, they can provide a local means to recycle kitchen waste as a replacement for a portion of corn and soy in the diet, resulting in more sustainable and lower cost production of eggs and meat (Georganas et al., 2020; Truong et al., 2019; Zu Ermgassen et al., 2016). Thus, there is a need to evaluate the performance of backyard-type native chickens whose diet is composed in part or entirely of kitchen waste. The local Naked Neck breed was superior when reared under various systems of feeding and housing (Garces et al., 2001). Keeping this in view, the present experiment was undertaken to explore the carcass traits, meat quality, and sensory attributes of Naked Neck chickens under two production systems and five nutritional regimens.

\section{Materials and Methods}

The care and use of the experimental birds were in accordance with the laws and regulations of Pakistan as affirmed by the Committee of Ethical Handling of Experimental Birds, University of Veterinary and Animal Sciences, Lahore, Pakistan (No. DR/758). This study was conducted at the Department of Poultry Production, University of Veterinary and Animal Sciences, A-Block, Ravi Campus, Pattoki, Pakistan. Pattoki is located at $31^{\circ} 1^{\prime} 0^{\prime \prime} \mathrm{N}, 73^{\circ} 50^{\prime} 60^{\prime \prime} \mathrm{E}$ at an elevation of $186 \mathrm{~m}$. The climate is hot and muggy with temperatures extending from $13^{\circ} \mathrm{C}$ in winter to $45^{\circ} \mathrm{C}$ in summer.

A total of 900 Naked Neck chicks $(400 \pm 27 \mathrm{~g}$ ) were picked from stock at random at the age of six weeks and divided into 10 treatment groups with 5 replicates of 18 birds each. A $2 \times 5$ factorial arrangement of treatments was applied according to a completely randomized design. Treatments consisted of two production systems (intensive and free-range) and five nutritional regimens, namely i) $100 \%$ commercial feed, ii) $75 \%$ commercial feed plus $25 \%$ kitchen waste, iii) $50 \%$ commercial feed plus kitchen waste, iv) $25 \%$ commercial feed plus $75 \%$ kitchen waste, and v) $100 \%$ kitchen waste.

The experimental birds were labelled individually and kept in a well-ventilated open-sided poultry house $(6.1 \mathrm{~m} \times 6.1 \mathrm{~m} \times 3.66 \mathrm{~m})$, oriented east to west, and were fed commercial grower ration according to the recommendations of the NRC (1994). Their weekly feed allowance was increased in correlation with their growing pattern. A stocking density of $0.06 \mathrm{~m}^{2}$ per bird and nipple drinking system at 10 birds per nipple were used as an intensive system till six weeks old. With the progression in age, stocking density was adjusted to a maximum of $0.14 \mathrm{~m}^{2}$ per bird.

For a free-range system, a pen measuring $11 \mathrm{~m}^{2}$ indoor area and $19 \mathrm{~m}^{2}$ for outdoor access were provided to 20 birds at $2 \mathrm{~m}^{2} /$ bird (Ghayas et al., 2020). Drinking water was provided with a nipple drinking system in the indoor area and supplementary feeders and drinkers were placed at 10 birds per feeder and 15 birds per drinker in the outdoor area.

The birds in both systems were fed kitchen waste and commercial poultry feed (Table 2) in a measured amount to calculate feed intake. The kitchen waste was collected from university student hostels and cafeterias of $A$ and C Block of UVAS Ravi Campus Pattoki, Pakistan. The restaurants in the vicinity of Ravi Campus were also utilized to obtain free kitchen waste. The kitchen waste was $35.6 \%$ dry matter with a proximate analysis (as fed) of $16.5 \%$ crude protein, $18.0 \%$ ether extract, and $6.0 \%$ ash. The commercial poultry feed is described in Table 1.

At eighteen weeks old, three Naked Neck cockerels (1625 $\pm 70 \mathrm{~g})$ were randomly selected from each replicate (total 150 cockerels) and slaughtered. These birds were weighed individually with an electrical balance (Wei Heng, Guangdong, China). All birds fasted for five hours before slaughter to keep their intestines and crop free of undigested feed (feed withdrawal period). The birds were slaughtered manually and humanely following the Halal method to ensure complete bleeding. Birds were de-feathered manually after slaughter, then eviscerated. After that, carcasses were soaked in cold water for one hour. Then the carcasses were hung to drain, and cut up into parts for further examination. The calculations of the yield of parameters were based on the percentage of live bodyweight at the time of slaughter (Raphulu et al., 2015). 
Table 1 Ingredient and nutrient composition of commercial feed for Naked Neck chickens between 7 and 18 weeks old

\begin{tabular}{lclc}
\hline Ingredients & $\%$ & \multicolumn{2}{c}{ Nutrients } \\
\hline Corn & 61.55 & Dry matter, \% & 89.50 \\
Soybean meal & 31.70 & Crude protein, \% & 20.02 \\
Soybean oil & 3.00 & Metabolizable energy, Kcal/kg & 3020 \\
Di-calcium phosphate & 1.70 & Calcium, \% & 0.91 \\
$\mathrm{NaCl}$ & 0.30 & Phosphorus, \% & 0.35 \\
Methionine & 0.12 & Lysine, \% & 1.09 \\
& & Methionine, \% & 0.43 \\
\hline
\end{tabular}

The hot carcass (without skin and giblets) was weighed before complete bleeding, de-feathering, and eviscerating to record dressed weight, which was then used to calculate carcass yield as:

$$
\text { Carcass yield } \%=\frac{\text { Dressed weight }(\mathrm{g})}{\text { Live weight }(\mathrm{g})} \times 100
$$

Each carcass was cut into prime parts (breast, thigh, drumstick, wings ribs \& back and their relative weight was worked out using this equation:

$$
\text { Carcass part } \%=\frac{\text { carcass part weight }(\mathrm{g})}{\text { carcass weight }(\mathrm{g})} \times 100
$$

Meat $\mathrm{pH}$ values were recorded after 15 minutes, 2 hours, and then 24 hours (ultimate $\mathrm{pH}$ ) of slaughter using a German pH meter (Thermo Fisher Scientific, Inc., Göteborg, Sweden). Approximately four hours after slaughter, the breast and thigh meat samples were separated and colour values (lightness, redness, yellowness) $\left(L^{*}, a^{*}, b^{*}\right)$ were recorded with a chromameter. The samples were kept in plastic bags for 24 hours at $8-10 \stackrel{\circ}{ } \mathrm{C}$ while hung up to dry, and then again weighed to estimate drip loss (Honikel, 1987). At that point samples were put away for 24 hours at $5 \stackrel{\circ}{ } \mathrm{C}$, then two cylindrical samples were taken from each breast, parallel to the grain, $12 \mathrm{~mm}$ broad, and at least $03 \mathrm{~cm}$ long to test shear force with a Warner-Bratzler (TAXT Plus, USA) shear force texture analyser (Stadig et al., 2016).

A sensory panel test was performed to evaluate the colour, aroma, taste, flavour, juiciness, tenderness, and overall acceptability of the samples. For sensory evaluation, samples of breast meat $(n=$ 150) were cooked to $72{ }^{\circ} \mathrm{C}$ without salt or spices (Castellini et al., 2002). Immediately after cooking, the samples were cut into pieces and presented to ten faculty staff, postgraduate students, and industry-linked personnel, who served as panellists. The panellists were taught how to assess each sensory attribute before the samples were served. Then, a consumer acceptance test was conducted based on a nine-point hedonic scale (Table 2).

Table 2 Description of hedonic scale used to evaluate meat from Naked Neck chickens fed varying levels of kitchen waste

\begin{tabular}{clclll}
\hline Score & Description & Score & Description & Score & Description \\
\hline 1 & Dislike extremely & 4 & Dislike slightly & 7 & Like moderately \\
2 & Dislike very much & 5 & Neither like nor dislike & 8 & Like very much \\
3 & Dislike moderately & 6 & Like slightly & 9 & Like extremely \\
\hline
\end{tabular}


Effects of production system and feeding regimen on carcass and meat quality characteristics and sensory attributes were determined through factorial ANOVA (SAS Institute Inc., Cary, North Carolina, USA). Treatment means for the significant effects were compared through Duncan's (1955) procedure for multiple comparisons, considering the difference to be real at probability $P=0.05$.

\section{Results and Discussion}

Meat quality attributes of the Naked Neck chickens were not influenced by kitchen waste, and the birds performed equally well in the free-range and intensive rearing systems. The effects of production system and dietary treatment on carcass and meat quality traits are shown in Tables 3, 4, 5, 6, and 7. Liver weight was higher in intensively reared birds than in free-range birds. A possible explanation for larger liver mass in birds reared under an intensive housing system is lack of exercise with fewer nutrients being expended for energy and thus the high energy diet stimulated hepatic lipogenesis. These findings are consistent with those of Hanyani (2012) and Zhao et al. (2012, 2015), who reported that chickens raised in cages had heavier carcasses and livers than those reared on the floor. In addition, Hrncar et al. (2014) reported higher liver weights in ducks reared in cages than those of deep litter floor systems. However, Ahmad et al. (2019) reported a contradictory result in which liver weight in Naked Neck chicken increased when the bids were reared under a semi-intensive system.

The birds fed on $100 \%$ kitchen waste had a remarkably high gizzard weight compared with those fed $75 \%, 50 \%, 25 \%$, and $0 \%$. The gizzard is the principal physical food processing organ of food in avian species and the relative size of the gizzard has been reported to increase with increased particle size in the diet (Amerah et al., 2007). The birds fed 100\% kitchen waste had more variation in the particle sizes of their foodstuffs, which was theorized to have increased the volume of gizzard and caused its walls to become thicker. This corresponded with the findings of de Verdal et al. (2010) that the gizzard became heavier with more forage in the diet. Likewise, Batkowska et al. (2015) observed increased gizzard weight in three chicken genotypes when they were fed a high-fibre diet containing wheat bran, crushed wheat, and green fodder. However, Farghly et al. (2019) did not detect differences in gizzard weight of commercial broilers that were fed ad libitum, were feed restricted, or were fed intermittently.

The meat from birds fed $100 \%$ kitchen waste was more yellow than meat from birds in the other treatment groups. This yellowness might result from the kitchen waste containing more carotenoids, xanthophyll, or related pigments. Da Silva et al. (2017) reported higher yellowness in the meat of slowgrowing chickens reared in the free-range system and provided with forages rich in carotenoids. More generally, a change of nutritional strategies has been reported to ultimately affect the colour of chicken meat (Batool et al., 2018). Hoffman et al. (2010) reported that nutrient quantity and quality, scavenging, and alternative feed resources may influence the meat colour of the chickens. Likewise, Fanatico et al. (2005) and Wattanachant (2008) observed that the colour of meat from indigenous chickens depended on the feeds that were provided.

Significant interactions between the rearing system and percentage of dietary kitchen waste were found for carcass yield, muscle $\mathrm{pH}$ at two hours post mortem, and overall acceptability. The birds reared intensively with a diet composed of $100 \%$ kitchen waste and those reared under a free-range system with commercial feed had the highest carcass yield, however, birds fed on $25 \%$ and $75 \%$ kitchen waste and reared under free-range system had the moderate carcass yield. Moreover, free-range birds fed with $50 \%$ or $100 \%$ kitchen waste and intensive birds fed $0 \%$ or $25 \%$ kitchen waste were the lowest in terms of carcass yield. These inconsistent results might be seen as being somewhat similar to the variation observed among other studies. Ying et al. (2011) reported that eviscerated carcass yield and proportions of breast and leg muscles were influenced by production system. Darwish et al. (2017) noted a higher percentage of carcass yield of broiler birds reared on the floor system. Sogunle et al. (2008) reported that the birds raised on the floor had higher dressing percentage than those raised in battery cages. Moreover, Hanyani (2012) reported that birds reared under semi-scavenging systems had higher slaughter and carcass weights than those of full-time scavenging birds (Kondombo, 2005; Mekonnen et al. 2010; da Silva et al., 2017). However, Poltowicz and Doktor (2011) indicated that carcass yield was unaffected by rearing system.

Breast meat from birds fed on $50 \%$ and $75 \%$ kitchen waste had high pH at two hours post mortem, whereas birds reared intensively with $25 \%$ kitchen waste and free-range birds without kitchen waste had lower $\mathrm{pH}$ values. Apparently, birds that received a diet with $50 \%$ commercial feed and $50 \%$ kitchen waste had better post-slaughter properties and their carcasses achieved the ideal ultimate $\mathrm{pH}$ quicker than other treatment groups. Ying et al. (2011) and Stadig et al. (2016) observed that meat pH depended largely on the feeding habits of the birds. Hanyani (2012) reported that chickens reared under semi-scavenging systems had better meat ultimate $\mathrm{pH}$ because their diet was rich in carotenoids. 
Table 3 Effect of production system and level of dietary kitchen waste on carcass characteristics of male Naked Neck chickens

\begin{tabular}{|c|c|c|c|c|c|c|c|}
\hline Production system & Feeding regimen & Live weight, $g$ & Carcass weight, g & Carcass yield, $\%$ & Breast meat yield, $\mathrm{g}$ & Thigh yield, $g$ & Drumstick yield, $\mathrm{g}$ \\
\hline Free-range & & 1630.00 & 1045.25 & 64.21 & 159.60 & 158.00 & 112.00 \\
\hline \multirow[t]{6}{*}{ Intensive } & & 1617.50 & 1058.75 & 65.63 & 161.60 & 154.00 & 103.00 \\
\hline & $0 \% \mathrm{KW}$ & 1653.75 & 1097.13 & 66.55 & 160.75 & 168.75 & 116.25 \\
\hline & $25 \% \mathrm{KW}$ & 1736.25 & 1101.25 & 63.49 & 183.25 & 183.75 & 120.00 \\
\hline & $50 \% \mathrm{KW}$ & 1605.00 & 985.63 & 61.63 & 147.00 & 147.50 & 108.75 \\
\hline & $75 \% \mathrm{KW}$ & 1535.00 & 1034.75 & 67.33 & 153.75 & 128.75 & 92.50 \\
\hline & $100 \% \mathrm{KW}$ & 1588.75 & 1041.25 & 65.60 & 158.25 & 151.25 & 151.25 \\
\hline \multirow[t]{5}{*}{ Free-range } & $0 \% \mathrm{KW}$ & 1632.50 & 1149.25 & $70.51^{a}$ & 164.00 & 165.00 & 130.00 \\
\hline & $25 \% \mathrm{KW}$ & 1732.50 & 1122.50 & $64.97^{\mathrm{abc}}$ & 169.00 & 187.50 & 130.00 \\
\hline & $50 \% \mathrm{KW}$ & 1710.00 & 1035.00 & $60.60^{\mathrm{C}}$ & 152.50 & 160.00 & 102.50 \\
\hline & $75 \% \mathrm{KW}$ & 1475.00 & 952.00 & $64.58^{\mathrm{abc}}$ & 156.00 & 127.50 & 82.50 \\
\hline & $100 \% \mathrm{KW}$ & 1600.00 & 967.50 & $60.38^{\mathrm{C}}$ & 156.50 & 150.00 & 115.00 \\
\hline \multirow[t]{5}{*}{ Intensive } & $0 \% \mathrm{KW}$ & 1675.00 & 1045.00 & $62.59^{c}$ & 157.50 & 172.50 & 102.50 \\
\hline & $25 \% \mathrm{KW}$ & 1740.00 & 1080.00 & $62.00^{\mathrm{C}}$ & 197.50 & 180.00 & 110.00 \\
\hline & $50 \% \mathrm{KW}$ & 1500.00 & 936.25 & $62.66^{\mathrm{bc}}$ & 141.50 & 135.00 & 115.00 \\
\hline & $75 \% \mathrm{KW}$ & 1595.00 & 1117.50 & $70.07^{\mathrm{ab}}$ & 151.50 & 130.00 & 102.50 \\
\hline & $100 \% \mathrm{KW}$ & 1577.50 & 1115.00 & $70.81^{a}$ & 160.00 & 152.50 & 85.00 \\
\hline \multicolumn{2}{|l|}{ Source of variation } & \multicolumn{6}{|c|}{ Probability effect $\neq 0$} \\
\hline \multicolumn{2}{|c|}{ Production system } & 0.8211 & 0.7272 & 0.3728 & 0.7963 & 0.7962 & 0.2158 \\
\hline \multicolumn{2}{|c|}{ Feeding regimen } & 0.2181 & 0.3065 & 0.1601 & 0.0599 & 0.2266 & 0.1146 \\
\hline \multicolumn{2}{|c|}{ Production system $\times$ feeding regimen } & 0.4228 & 0.0718 & 0.0084 & 0.5120 & 0.9651 & 0.0955 \\
\hline \multicolumn{2}{|l|}{ RMSE } & 27.82 & 20.55 & 0.93 & 4.06 & 7.44 & 3.96 \\
\hline
\end{tabular}

${ }^{a, b, c}$ Within a column, means with a common superscript were not different with probability $P=0.05$

RMSE: root mean square error, KW: kitchen waste 
Table 4 Effect of production system and level of dietary kitchen waste on cut-up parts of male Naked Neck chickens

\begin{tabular}{|c|c|c|c|c|c|c|c|}
\hline Production system & Feeding regimen & Heart, g & Wings, $g$ & Ribs \& Back, g & Liver, $g$ & Gizzard, g & Abdominal fat, $g$ \\
\hline Free-range & & 9.30 & 106.50 & 255.00 & $24.25^{\mathrm{b}}$ & 26.60 & 2.65 \\
\hline \multirow[t]{6}{*}{ Intensive } & & 9.45 & 107.00 & 242.00 & $27.70^{\mathrm{a}}$ & 28.95 & 2.95 \\
\hline & $0 \% \mathrm{KW}$ & 9.00 & 108.75 & 260.00 & 23.25 & $25.88^{\mathrm{b}}$ & 2.88 \\
\hline & $25 \% \mathrm{KW}$ & 9.88 & 112.50 & 257.50 & 28.75 & $27.50^{\mathrm{b}}$ & 2.88 \\
\hline & $50 \% \mathrm{KW}$ & 9.00 & 101.25 & 255.00 & 26.38 & $25.13^{b}$ & 2.75 \\
\hline & $75 \% \mathrm{KW}$ & 9.13 & 97.50 & 240.00 & 25.75 & $27.25^{\mathrm{b}}$ & 2.88 \\
\hline & $100 \% \mathrm{KW}$ & 9.88 & 113.75 & 230.00 & 25.75 & $33.13^{\mathrm{a}}$ & 2.63 \\
\hline \multirow[t]{5}{*}{ Free-range } & $0 \% \mathrm{KW}$ & 9.75 & 105.00 & 265.00 & 21.75 & 24.75 & 2.75 \\
\hline & $25 \% \mathrm{KW}$ & 8.25 & 105.00 & 285.00 & 25.75 & 25.50 & 2.25 \\
\hline & $50 \% \mathrm{KW}$ & 9.50 & 112.50 & 265.00 & 26.25 & 25.25 & 2.75 \\
\hline & $75 \% \mathrm{KW}$ & 8.75 & 100.00 & 225.00 & 24.00 & 25.25 & 3.25 \\
\hline & $100 \% \mathrm{KW}$ & 10.25 & 110.00 & 235.00 & 23.50 & 32.25 & 2.25 \\
\hline \multirow[t]{5}{*}{ Intensive } & $0 \% \mathrm{KW}$ & 8.25 & 112.50 & 255.00 & 24.75 & 27.00 & 3.00 \\
\hline & $25 \% \mathrm{KW}$ & 11.50 & 120.00 & 230.00 & 31.75 & 29.50 & 3.50 \\
\hline & $50 \% \mathrm{KW}$ & 8.50 & 90.00 & 245.00 & 26.50 & 25.00 & 2.75 \\
\hline & $75 \% \mathrm{KW}$ & 9.50 & 95.00 & 255.00 & 27.50 & 29.25 & 2.50 \\
\hline & $100 \% \mathrm{KW}$ & 9.50 & 117.50 & 225.00 & 28.00 & 34.00 & 3.00 \\
\hline \multicolumn{2}{|l|}{ Source of variation } & \multicolumn{6}{|c|}{ Probability effect $\neq 0$} \\
\hline \multicolumn{2}{|c|}{ Production system } & 0.8328 & 0.9424 & 0.1623 & 0.0391 & 0.0889 & 0.2602 \\
\hline \multicolumn{2}{|c|}{ Feeding regimen } & 0.8486 & 0.5005 & 0.1931 & 0.3295 & 0.0062 & 0.9632 \\
\hline \multicolumn{2}{|c|}{ Production system $\times$ feeding regimen } & 0.2286 & 0.4646 & 0.0889 & 0.8398 & 0.8405 & 0.1799 \\
\hline \multicolumn{2}{|l|}{ RMSE } & 0.34 & 3.35 & 5.02 & 0.82 & 0.77 & 0.13 \\
\hline
\end{tabular}

${ }^{a, b, c}$ Within a column, means with a common superscript were not different with probability $P=0.05$

RMSE: root mean square error, KW: kitchen waste 
Table 5 Effect of production system and level of dietary kitchen waste regimen on meat colour of male Naked Neck chickens

\begin{tabular}{|c|c|c|c|c|c|c|}
\hline Production system & Feeding regimen & Lightness, L* & Redness, $\mathrm{a}^{*}$ & Yellowness, b* & Chroma, $c$ & Hue angle, $h$ \\
\hline Free-range & & 51.03 & 13.59 & 8.67 & 16.39 & 31.66 \\
\hline \multirow[t]{6}{*}{ Intensive } & & 51.91 & 12.78 & 7.80 & 15.30 & 30.60 \\
\hline & $0 \% \mathrm{KW}$ & 50.95 & 13.80 & $9.52^{b}$ & 16.67 & 34.53 \\
\hline & $25 \% \mathrm{KW}$ & 52.13 & 13.63 & $8.50^{a b c}$ & 18.26 & 32.55 \\
\hline & $50 \% \mathrm{KW}$ & 50.28 & 12.80 & $7.04^{\mathrm{bc}}$ & 14.63 & 28.64 \\
\hline & $75 \% \mathrm{KW}$ & 51.92 & 12.25 & $10.54^{\mathrm{a}}$ & 16.38 & 38.19 \\
\hline & $100 \% \mathrm{KW}$ & 52.05 & 13.44 & $5.57^{\mathrm{C}}$ & 15.29 & 21.77 \\
\hline \multirow[t]{5}{*}{ Free-range } & $0 \% \mathrm{KW}$ & 49.47 & 13.55 & 9.60 & 16.37 & 34.89 \\
\hline & $25 \% \mathrm{KW}$ & 48.59 & 16.49 & 8.85 & 18.76 & 27.56 \\
\hline & $50 \% \mathrm{KW}$ & 52.06 & 12.44 & 7.53 & 14.56 & 31.17 \\
\hline & $75 \% \mathrm{KW}$ & 51.87 & 12.22 & 10.76 & 16.46 & 38.37 \\
\hline & $100 \% \mathrm{KW}$ & 53.15 & 13.24 & 6.63 & 15.82 & 26.34 \\
\hline \multirow[t]{5}{*}{ Intensive } & $0 \% \mathrm{KW}$ & 52.44 & 14.06 & 9.45 & 16.97 & 34.17 \\
\hline & $25 \% \mathrm{KW}$ & 55.68 & 10.77 & 8.16 & 13.76 & 37.53 \\
\hline & $50 \% \mathrm{KW}$ & 48.50 & 13.15 & 6.55 & 14.70 & 26.10 \\
\hline & $75 \% \mathrm{KW}$ & 51.98 & 12.27 & 10.33 & 16.30 & 38.02 \\
\hline & $100 \% \mathrm{KW}$ & 50.94 & 13.65 & 4.51 & 14.76 & 17.19 \\
\hline \multicolumn{2}{|l|}{ Source of variation } & \multicolumn{5}{|c|}{ Probability effect $\neq 0$} \\
\hline \multicolumn{2}{|c|}{ Production system } & 0.5919 & 0.2728 & 0.3375 & 0.1493 & 0.7499 \\
\hline \multicolumn{2}{|c|}{ Feeding regimen } & 0.9263 & 0.6168 & 0.0309 & 0.3729 & 0.0725 \\
\hline \multicolumn{2}{|c|}{ Production system $\times$ feeding regimen } & 0.2904 & 0.0661 & 0.9566 & 0.1581 & 0.4653 \\
\hline \multicolumn{2}{|l|}{ RMSE } & 0.75 & 0.41 & 0.53 & 0.41 & 1.89 \\
\hline
\end{tabular}

${ }^{a, b, c}$ Within a column, means with a common superscript were not different with probability $P=0.05$

RMSE: root mean square error, KW: kitchen waste 
Table 6 Effect of production system and level of dietary kitchen waste on meat quality attributes of male Naked Neck chickens

\begin{tabular}{|c|c|c|c|c|c|c|c|}
\hline Production system & Feeding regimen & Cooking Loss,\% & Drip Loss,\% & Shear force, $\mathrm{N}$ & $\mathrm{pH}, 15 \mathrm{~min}$ & $\mathrm{pH}, 2 \mathrm{hr}$ & $\mathrm{pH}, 24 \mathrm{hr}$ \\
\hline Free-range & & 33.32 & 3.27 & 28.35 & 6.92 & 6.74 & 6.11 \\
\hline \multirow[t]{6}{*}{ Intensive } & & 31.32 & 3.44 & 23.67 & 6.95 & 6.73 & 6.12 \\
\hline & $0 \% \mathrm{KW}$ & 33.93 & 2.48 & 28.95 & 6.82 & $6.55^{\mathrm{b}}$ & 6.11 \\
\hline & $25 \% \mathrm{KW}$ & 30.75 & 3.71 & 24.21 & 6.96 & $6.96^{\mathrm{ab}}$ & 6.12 \\
\hline & $50 \% \mathrm{KW}$ & 30.75 & 4.40 & 21.90 & 7.09 & $6.90^{\mathrm{a}}$ & 6.06 \\
\hline & $75 \% \mathrm{KW}$ & 33.26 & 2.61 & 28.12 & 6.98 & $6.87^{\mathrm{a}}$ & 6.12 \\
\hline & $100 \% \mathrm{KW}$ & 33.04 & 3.57 & 26.88 & 6.82 & $6.67^{\mathrm{ab}}$ & 6.16 \\
\hline \multirow[t]{5}{*}{ Free-range } & $0 \% \mathrm{KW}$ & 33.94 & 2.44 & 30.68 & 6.75 & $6.51^{\mathrm{c}}$ & 6.19 \\
\hline & $25 \% \mathrm{KW}$ & 33.57 & 3.15 & 24.92 & 6.95 & $6.93^{\mathrm{ab}}$ & 6.15 \\
\hline & $50 \% \mathrm{KW}$ & 33.00 & 3.96 & 19.09 & 7.10 & $6.56^{\mathrm{bc}}$ & 5.89 \\
\hline & $75 \% \mathrm{KW}$ & 33.05 & 3.48 & 35.52 & 6.97 & $6.95^{\mathrm{ab}}$ & 6.05 \\
\hline & $100 \% \mathrm{KW}$ & 33.06 & 3.33 & 31.55 & 6.84 & $6.74^{\mathrm{bc}}$ & 6.26 \\
\hline \multirow[t]{5}{*}{ Intensive } & $0 \% \mathrm{KW}$ & 33.93 & 2.53 & 27.22 & 6.90 & $6.58^{\mathrm{bc}}$ & 6.03 \\
\hline & $25 \% \mathrm{KW}$ & 27.93 & 4.28 & 23.50 & 6.97 & $6.45^{\mathrm{C}}$ & 6.10 \\
\hline & $50 \% \mathrm{KW}$ & 33.53 & 4.85 & 24.72 & 7.09 & $7.24^{\mathrm{a}}$ & 6.24 \\
\hline & $75 \% \mathrm{KW}$ & 28.19 & 1.73 & 20.73 & 6.98 & $6.79^{b c}$ & 6.19 \\
\hline & $100 \% \mathrm{KW}$ & 33.03 & 3.81 & 22.20 & 6.81 & $6.61^{\mathrm{bc}}$ & 6.07 \\
\hline \multicolumn{2}{|l|}{ Source of variation } & \multicolumn{6}{|c|}{ Probability effect $\neq 0$} \\
\hline \multicolumn{2}{|c|}{ Production system } & 0.1063 & 0.8144 & 0.1196 & 0.7285 & 0.9747 & 0.8568 \\
\hline \multicolumn{2}{|c|}{ Feeding regimen } & 0.2830 & 0.4278 & 0.5273 & 0.1637 & 0.0411 & 0.9772 \\
\hline \multicolumn{2}{|c|}{ Production system $\times$ feeding regimen } & 0.3034 & 0.7146 & 0.2501 & 0.9532 & 0.0012 & 0.3622 \\
\hline \multicolumn{2}{|c|}{ RMSE } & 0.64 & 0.33 & 1.51 & 0.04 & 0.05 & 0.04 \\
\hline
\end{tabular}

${ }^{a, b, c}$ Within a column, means with a common superscript were not different with probability $P=0.05$ RMSE: root mean square error, KW: kitchen waste 
Table 7 Effect of production system and level of dietary kitchen waste on meat sensory attributes of male Naked Neckchicken

\begin{tabular}{|c|c|c|c|c|c|c|c|c|}
\hline Production system & Feeding regimen & Colour & Aroma & Taste & Flavour & Juiciness & Tenderness & Overall acceptability \\
\hline Free-range & & 6.11 & 5.63 & 6.00 & 6.60 & 5.74 & 5.71 & 6.26 \\
\hline \multirow[t]{6}{*}{ Intensive } & & 5.97 & 5.26 & 5.80 & 6.57 & 6.57 & 5.34 & 6.00 \\
\hline & $0 \% \mathrm{KW}$ & 5.53 & 5.26 & 6.00 & 6.21 & 5.47 & 5.74 & 6.00 \\
\hline & $25 \% \mathrm{KW}$ & 6.11 & 5.67 & 5.44 & 6.78 & 5.89 & 5.11 & 5.67 \\
\hline & $50 \% \mathrm{KW}$ & 6.64 & 5.36 & 6.57 & 6.29 & 5.50 & 5.43 & 6.14 \\
\hline & $75 \% \mathrm{KW}$ & 5.79 & 5.07 & 5.79 & 6.36 & 5.36 & 5.14 & 6.43 \\
\hline & $100 \% \mathrm{KW}$ & 6.36 & 6.00 & 5.50 & 7.50 & 6.36 & 6.00 & 6.29 \\
\hline \multirow[t]{5}{*}{ Free-range } & $0 \% \mathrm{KW}$ & 5.71 & 4.71 & 5.29 & 6.14 & 5.71 & 5.29 & $7.14^{a}$ \\
\hline & $25 \% \mathrm{KW}$ & 5.71 & 6.14 & 6.29 & 6.29 & 5.43 & 5.86 & $4.86^{b}$ \\
\hline & $50 \% \mathrm{KW}$ & 7.14 & 5.43 & 7.14 & 6.14 & 6.00 & 6.00 & $7.14^{\mathrm{a}}$ \\
\hline & $75 \% \mathrm{KW}$ & 5.71 & 5.57 & 5.29 & 6.57 & 5.43 & 5.43 & $5.57^{\mathrm{ab}}$ \\
\hline & $100 \% \mathrm{KW}$ & 6.29 & 6.29 & 6.00 & 7.86 & 6.14 & 6.00 & $6.57^{\mathrm{ab}}$ \\
\hline \multirow[t]{5}{*}{ Intensive } & $0 \% \mathrm{KW}$ & 5.00 & 5.14 & 6.14 & 5.86 & 5.43 & 5.86 & $6.00^{\mathrm{ab}}$ \\
\hline & $25 \% \mathrm{KW}$ & 6.43 & 5.57 & 5.57 & 7.29 & 5.86 & 5.14 & $5.57^{\mathrm{ab}}$ \\
\hline & $50 \% \mathrm{KW}$ & 6.14 & 5.29 & 6.00 & 6.43 & 5.00 & 4.86 & $5.14^{\mathrm{b}}$ \\
\hline & $75 \% \mathrm{KW}$ & 5.86 & 4.57 & 6.29 & 6.14 & 5.29 & 4.86 & $7.29^{\mathrm{a}}$ \\
\hline & $100 \% \mathrm{KW}$ & 6.43 & 5.71 & 5.00 & 7.14 & 6.57 & 6.00 & $6.00^{\mathrm{ab}}$ \\
\hline \multicolumn{2}{|l|}{ Source of variation } & \multicolumn{7}{|c|}{ Probability effect $\neq 0$} \\
\hline \multicolumn{2}{|c|}{ Production system } & 0.9508 & 0.3155 & 0.8762 & 0.6869 & 0.6535 & 0.5526 & 0.5186 \\
\hline \multicolumn{2}{|c|}{ Feeding regimen } & 0.1578 & 0.6375 & 0.4104 & 0.1637 & 0.4299 & 0.6557 & 0.8887 \\
\hline \multicolumn{2}{|c|}{ Production system $\times$ feeding regimen } & 0.3928 & 0.9662 & 0.3808 & 0.3534 & 0.8237 & 0.8379 & 0.0367 \\
\hline \multicolumn{2}{|c|}{ RMSE } & 0.18 & 0.20 & 0.20 & 0.20 & 0.18 & 0.20 & 0.19 \\
\hline
\end{tabular}

${ }^{a, b, c}$ Within a column, means with a common superscript were not different with probability $P=0.05$

RMSE: root mean square error, KW: kitchen waste 
Meat from birds fed $75 \%$ kitchen waste in the intensive system and free-range birds fed on $0 \%$ and $25 \%$ kitchen waste were deemed by the panellists to have higher overall meat acceptability, whereas meat from the free-range birds fed $25 \%$ kitchen waste had the lowest score for overall acceptability. Similarly, Hanyani (2012) reported that consumers preferred meat from birds reared under a semi-scavenging system to that from full scavenging chickens. Furthermore, these consumers noted significant differences between the two systems in meat juiciness, impression on the first bite, and typical taste and flavour intensity. Mikulski et al. (2011) reported that the colour of thigh and breast muscles was significantly darker when birds were reared in an open house system compared with those reared indoors. Farghly et al. (2019) did not detect differences in the sensory attributes of meat from commercial broilers that were fed ad-libitum, feed restricted, or intermittently.

\section{Conclusions}

Naked Neck chickens produced meat of equivalent value, whether produced under the intensive or the free-range system and irrespective of the level of kitchen waste that was fed. Adopting the practice of feeding kitchen waste to backyard chickens may lead to improved food security.

\section{Acknowledgments}

The authors gratefully acknowledge the cooperation extended by Dr. Sohail Ahmad and Dr. Muhammad Usman for their guidance in and facilitation of this research.

\section{Author's Contributions}

EB conducted this study as part of his Ph.D. research under the supervision of $\mathrm{JH}, \mathrm{AM}$, and AK. AM and $\mathrm{KH}$ helped in reviewing the manuscript. $\mathrm{JH}$ helped in the statistical analysis and formatting the manuscript.

\section{Conflict of Interest Declaration}

The authors have no potential conflicts of interest.

\section{References}

Ahmad, S., Mahmud, A., Hussain, J. \& Javed, K., 2019. Morphological and carcass traits of three chicken genotypes under free-range, semi-intensive, and intensive housing systems. Turk. J. Vet. Anim. Sci. 43(3), 342-352. DOI: 10.3906/vet-1902-9

Amerah, A. M., Lentle, R. G. \& Ravindran, V., 2007. Influence of feed form on gizzard morphology and particle size spectra of duodenal digesta in broiler chickens. J. Poult. Sci. 44(2), 175-181. DOI: 10.2141/jpsa.44.175

Ajayi, F.O., 2010. Nigerian indigenous chicken: A valuable genetic resources for meat and egg production. Asian J. Poult. Sci. 4(4), 164-172. DOI: 10.3923/ajpsaj.2010.164.172

Batkowska, J., Brodacki A., Zi . eba, G., Horba'nczuk, J.O. \& Łukaszewicz, M., 2015. Growth performance, carcass traits and physical properties of chicken meat as affected by genotype and production system. Arch. Anim. Breed. 58(2), 325-333. DOI: 10.5194/aab-58-325-2015

Batool, T., Farooq, S., Roohi, N., Mahmud, A., Usman, M., Ghayas, A. \& Ahmad, S., 2018. Effect of different dietary lysine regimens on meat quality attributes in varieties of indigenous Aseel chicken. Kafkas Univ. Vet. Fak. Derg. 24(5), 639-645. DOI: 10.9775/kvfd.2018.19523

Castellini, C., Mugnai, C. \& Dal Bosco, A., 2002. Effect of organic production system on broiler carcass and meat quality. Meat Sci. 60(3), 219-225. DOI: 10.1016/S0309-1740(01)00124-3

Chulayo, A.Y., Muchenje, V., Mwale, M. \& Masika, P.J. 2011. Effects of some medicinal plants on consumer sensory characteristics of village chicken meat. African J. Biotech. 10(31), 815- 820.

Darwish, A.H., El-Sayiad, G.A., El-Maghawry, A.M. \& Mahrose, K.M., 2017. Growth performance, carcass traits and some blood parameters of broiler chicks as affected by housing system. Zagazig J. Agric. Res. 44(4), 1379-1387.

de Verdal, H., Mignon-Grasteau, S., Jeulin, C., Le Bihan-Duval, E., Leconte, M., Mallet, S., Martin, C. \& Narcy, A., 2010. Digestive tract measurements and histological adaptation in broiler lines divergently selected for digestive efficiency. Poult. Sci. 89(9), 1955-1961. DOI: 10.3382/ps.2010-813

da Silva, D.C.F., de Arruda, A.M.V. \& Gonc,alves, A.A., 2017. Quality characteristics of broiler chicken meat from freerange and industrial poultry system for the consumers. J. Food Sci. Technol. 54(7), 1818-1826. DOI: $10.1007 / s 13197-017-2612-x$

Duncan, D.B., 1955. Multiple range and multiple $F$ tests. Biometrics. 11(1), 1-42. https://doi.org/10.2307/3001478

Elson, H. A., 2010. Poultry housing and husbandry. Br. Poult. Sci. 51(1), 23-34. DOI: 10.1080/00071668.2010.497300

ESOP (Economic Survey of Pakistan), 2020. Economic Survey of Pakistan 2019-20. Ministry of Finance, Finance Division, Government of Pakistan, Islamabad.

Fanatico, A. C., Pillai, P.B., Emmert, J.L. \& Owens, C.M., 2007. Meat quality of slow- and fast-growing chicken genotypes fed low-nutrient or standard diets and raised indoors or with outdoor access. Poult. Sci. 86(10), 22452255. DOI: $10.1093 / \mathrm{ps} / 86.10 .2245$

Fanatico, A.C., Pillai, P.B., Cavitt, L.C., Owens C.M. \& Emmert, J.L., 2005. Evaluation of slower-growing genotypes grown with and without outdoor access: growth performance and carcass yield. Poult. Sci. 84(8), 1321-1327. DOI: $10.1093 / \mathrm{ps} / 84.8 .1321$ 
FAO, 2009. Status and trends report on animal genetic resources-2008. In: information document CGRFA/WG-ANGR5/09/inf.7.Rome. httP://www.fao.

Farghly, M.F., Mahrose, K.M., Enas, Ahmad, A.M., Rehman, Z. U. \& Yu, S., 2019. Implementation of different feeding regimes and flashing light in broiler chicks. Poult. Sci. 98(5), 2034-2042. DOI: 10.3382/ps/pey577

Garces, A., Casey, N. \& Horst, P., 2001. Productive performance of naked neck, frizzle and dwarf laying hens under various natural climates and two nutritional treatments. S. Afr. J. Anim. Sci. 31, 174-180. DOI: 10.4314/sajas.v31i3.3800.

Georganas, A., Giamouri, E., Pappas, A.C., Papadomichelakis, G., Galliou, F., Manios, T., Tsiplakou, E., Fegeros, K. \& Zervas, G., 2020. Bioactive compounds in food waste: A review on the transformation of food waste to animal feed. Foods 9, 291. DOI: 10.3390/foods9030291

Ghayas, A., Hussain, J., Mahmud, A. \& Jaspal, M.H., 2020. Evaluation of three fast- and slow-growing chicken strains reared in two production environments. S. Afr. J. Anim. Sci. 50(3), 378-388. DOI: 10.4314/sajas.v50i3.4

Hanyani, C.T., 2012. Effect of full and semi-scavenging rearing systems on crop contents and the quality of meat from village chickens during the spring season of eastern cape, South Africa. MSc. thesis, Department of Livestock and Pasture Science, Faculty of Science and Agriculture, University of Fort Hare, South Africa.

Hoffman, L.C., Britz, T.J. \& Schnetler, D.C. 2010. Bruising on ostrich carcasses and the implications on the microbiology and the losses in utilisable meat when removing them post-evisceration or post-chilling. Meat Sci. 86 (1), $398-$ 404. DOI: 10.1016/j.meatsci.2010.05.024

Honikel, K.O., 1987. How to measure the water-holding capacity of meat? Recommendation of a standardized method. Evaluation and control of meat quality in pigs. The Hague, Netherlands, 129-142.

Hrncar, C., Weis, J., Petricova, L. \& Bujko, J., 2014. Effect of housing system, slaughter age and sex on slaughter and carcass parameters of broiler ducks. Anim. Sci. Biotechnol. 47(2), 254-257.

Jin, L. \& Craig, J.V., 1994. Some effects of cage and floor rearing on commercial white Leghorn pullets during growth and the first year on egg production. Poult. Sci. 67(10), 1400-1406. https://doi.org/10.3382/ps.0671400

Jin, S., Yang, L., Zang, H., Xu, Y., Chen, X., Chen, X., Liu, P. \& Geng, Z., 2019. Influence of free-range days on growth performance, carcass traits, meat quality, lymphoid organ indices, and blood biochemistry of Wannan Yellow chickens. Poult. Sci. 98(12), 6602- 6610. DOI: 10.3382/ps/pez504

Kondombo, S.R., 2005. Improvement of village chicken production in a mixed (chicken-ram) farming system in Burkina Faso. PhD thesis, Wageningen Institute of Animal Sciences, Animal Nutrition Group, Wageningen University, The Netherlands. 208.

Mekkonen, H., Mulatu, D., Kelay, B. \& Berhan, T. 2010. Assessment of the nutritional status of indigenous scavenging chickens in Ada'a district, Ethiopia. Trop. Anim. Health Prod. 42(1), 123-130. DOI: 10.1007/s11250-009-9395-7

Mikulski, D., Celej, J., Jankowski, J., Majewska, T. \& Mikulska, M., 2011. Growth Performance, Carcass Traits and Meat Quality of Slower-growing and Fast-growing Chickens Raised with and without Outdoor Access. Asian-Aust. J. Anim. Sci. 24(10), 1407-1416. DOI: 10.5713/ajas.2011.11038

Mueller, S., Kreuzer, M., Siegrist, M., Mannale, K., Messikommer, R.E. \& Gangnat, I.D.M., 2018. Carcass and meat quality of dual-purpose chickens (Lohmann Dual, Belgian Malines, Schweizerhuhn) in comparison to broiler and layer chicken types. Poult. Sci. 97(9), 3325-3336. DOI: 10.3382/ps/pey172

Ojedapo, L.O., 2013. Effect of two housing systems (cages vs deep litter) on external and internal egg characteristics of a commercial laying birds reared in derived savanna zone of Nigeria. Transitional J. Sci. Tech. 3(7), 25-34.

Olaniyi, O.A., Oyenaiya, O.A., Sogunle, O.M., Akinola, O.S., Adeyemi, O.A. \& Ladokun, O.A., 2012. Free range and deep litter housing systems: effect on performance and blood profile of two strains of cockerel chickens. Trop. Subtrop. Agroecosyst. 15(3), 511-523.

Padhi, M.K., 2016. Importance of indigenous breeds of chicken for rural economy and their improvements for higher production performance. Scientifica. 2604685(6), 1-9. DOI: 10.1155/2016/2604685

Połtowicz, K. \& Doktor, J., 2011. Effect of free-range raising on performance, carcass attributes and meat quality of broiler chickens. Anim. Sci. Pap. Rep. 29(2), 139-149.

Rahman, M.M., Faruque, S., Islam, M.S., Islam, M.N. \& Rashid, M.A., 2013. Comparison of growth performance and meat yield of Hilly Chicken under two feeding regimens. Agric. 11(2), 38-43. DOI: 10.3329/agric.v11i2.17485

Raphulu, T., Van Rensburg, C.J. \& Coertze, R.J., 2015. Carcass composition of Venda indigenous scavenging chickens under village management. J. Agric. Rural Develop. Trop. Subtrop. 116(1), 27-35.

Rajkumar, U., Haunshi, S., Paswan, C., Raju, M.V.L.N., Rama Rao, S.V. \& Chatterjee, R.N., 2017. Characterization of indigenous Aseel chicken breed for morphological, growth, production, and meat composition traits from India. Poult. Sci. 96(7), 2120-2126. DOI: 10.3382/ps/pew492

Rehman, M.S., Mahmud, A., Mehmood, S., Pasha, T.N., Hussain, J. \& Khan, M.T., 2017. Blood biochemistry and immune response in Aseel chicken under free range, semi-intensive, and confinement rearing systems. Poult. Sci. 96(1), 226-233. DOI: 10.3382/ps/pew278

Shahzad, M., Rizvi, F., Khan, A., Siddique, M., Khan, M.Z. \& Bukhari, S.M., 2011. Diagnosis of avain Paramyxovirus Type-1 infection in chicken by immunoflourescence technique. Int. J. Agric. Bio. 13(2), 266-270.

Sogunle, O.M., Egbeyale, L.T., Bajomo, T.T., Bamigboje, O.V. \& Fanimo, A.O., 2008. Comparison of the performance, carcass characteristics and haematological parameters of broiler chicks reared in cage and floor. Pak. J. Biol. Sci. 11(3), 480-483. DOI: 10.3923/pjbs.2008.480.483

Sow, T.M. \& Grongnet, J.F., 2010. Sensory characteristics and consumer preference for chicken meat in Guinea. Poult. Sci. 89(10), 2281-2292. https://doi.org/10.3382/ps.2010-00679 
Stadig, L.M., Rodenburg, T.B., Reubens, B., Aerts, J., Duquenne, B. \& Tuyttens, F.A.M., 2016. Effects of free- range access on production parameters and meat quality, composition and taste in slow growing broiler chickens. Poult. Sci. 95(12), 2971-2978. DOI: 10.3382/ps/pew226

Tougan, P.U., Dahouda, M., Ahounou, G.S., Salifou, C.F.A. \& Kpodekon, M.T., 2013a. Effect of breeding mode, type of muscle and slaughter age on technological meat quality of local poultry population of Gallus gallus species of Benin. Int. J. Biosci. 12(8), 81-97. DOI: 10.12692/ijb/3.5.108-116

Tougan, P.U., Dahouda, M., Salifou, C.F.A., Ahounou, G.S. \& Kpodekon, M.T., 2013b. Assessment of nutritional quality of meat of local poultry population of Gallus gallus species of Benin. J. Anim. Plant Sci. 19(2): 2908-2922.

Truong, L., Morash, D., Liu, Y., King, A., 2019. Food waste in animal feed with a focus on use for broilers. International J. Recycling Organic Waste Agric. 8, 417-429. https://doi.org/10.1007/s40093-019-0276-4

Wattanachant, S., 2008. Factors affecting the quality characteristics of Thai indigenous chicken meat. J. Sci. Tech. 15(4), 317-332.

Wichchukit, S. \& O'Mahony, M., 2014. The 9-point hedonic scale and hedonic ranking in food science: Some reappraisals and alternatives. J. Sci. Food Agri. 95(11), 2167-2178. DOI: 10.1002/jsfa.6993

Xiang, H., Chen, S., Zhang, H., Zhu, X., Wang, D., Liu, H., Wang, J., Yin, T., Liu, L., Kong, M., Zhang, J., Li, H. \& Zhao, $X ., 2018$. Transcriptome changes provide genetic insights into the effects of rearing systems on chicken welfare and product quality. J. Anim. Sci. 96(11), 4553-4561. DOI: 10.1093/jas/sky314

Ying, L., Luoa, C., Wanga, J. \& Guo, F., 2011. Effects of different raising systems on growth performance, carcass, and meat quality of medium-growing chickens. J. Appl. Anim. Res. 45(1), 326-330. https://doi.org/10.1080/09712119.2016.1190735

Yousif, I.A. \& Eltayeb, N.M., 2011. Performance of Sudanese native Dwarf and Bare Neck chicken raised under improved traditional production system. Agric. Bio. J. N. Am. 2(5), 860-866.

Youssao, A.K.I., Assogba, N.M., Alkoiret, T.I., Dahouda, M. \& Idrissou, N.D., 2012. Comparison of growth performance, carcass characteristics and sensory characters of Benin indigenous chickens and Label Rouge (T55 $\times$ SA51). Afr. J. Biotech. 11(89), 15569-15579. DOI: 10.5897/AJB11.1747

Youssao, A.K.I., Senou, M., Dahouda, M., Kpodekon, T.M. \& Jenontin, J., 2009. Genetic improvement of local chickens by crossing with the Label Rouge (T55XSA51): Growth performances and heterosis effects. Int. J. Poult. Sci. 8(6), 536-544. DOI: 10.3923/ijps.2009.536.544

Zhang, B., Fu, Z., Huang, J., Wang, J., Xu, S. \& Zhang, L., 2018. Consumer's perceptions, purchase intention, and willingness to pay a premium price for safe vegetables: a case study of Beijing, China. J. Clean. Prod. 197(1), 1498-1507. https://doi.org/10.1016/j.jclepro.2018.06.273

Zhao, X.L., Siegel, P.B., Liu, Y.P., Wang, Y., Gilbert, E.R., Zhu, Q. \& Zhang, L., 2012. Housing system affects broiler characteristics of local Chinese breed reciprocal crosses. Poult. Sci. 91(9), 2405-2410. DOI: 10.3382/ps.201202165

Zhao, X., Ren, W., Siegel, P.B., Li, J., Yin, H., Liu, Y., Wang, Y., Zhang, Y., Honaker, C.F. \& Zhu, Q., 2015. Housing systems interacting with sex and genetic line affect broiler growth and carcass traits. Poult. Sci. 94(7), 1711-1717. DOI: $10.3382 / p s / p e v 128$

Zu Ermgassen, E.K., Phalan, B., Green, R.E. \& Balmford, A., 2016. Reducing the land use of EU pork production: Where there's swill, there's a way. Food Policy 58, 35-48. DOI: 10.1016/j.foodpol.2015.11.001 